\title{
Successful treatment of Pseudomonas aeruginosa septicaemia and meningitis with neutropenia-the presenting feature of hypogammaglobulinaemia
}

\author{
P. G. B. JOHNSTON \\ M.B., M.R.C.P.
}

\author{
D. C. E. SPELleR* \\ B.M., M.R.C.P., M.R.C.Path.
}

\section{Departments of Paediatrics and *Clinical Bacteriology, Bristol Royal Hospital for Sick Children, St Michael's Hill, Bristol BS2 8BJ}

\begin{abstract}
Summary
A neutropenic child of 20 months suffered generalized infection with Pseudomonas aeruginosa, involving the bloodstream and the meninges. This was successfully treated with intravenous and intrathecal antibiotics and granulocyte transfusions before granulopoiesis recovered spontaneously. No immunoglobulin replacement was given during the acute episode, as the diagnosis of $\mathrm{X}$-linked hypogammaglobulinaemia was made subsequently.
\end{abstract}

\section{Introduction}

Gram-negative bacilli are only occasionally responsible for the serious infections which characterize untreated hypogammaglobulinaemia (Rosen and Janeway, 1966; Medical Research Council, 1971). Only one case presenting with Pseudomonas aeruginosa septicaemia has been reported (Speirs, Selwyn and Nicholson, 1963). Meningitis in hypogammaglobulinaemia is most frequently caused by Streptococcus pneumoniae or Neisseria meningitidis.

The morbidity and mortality of $P$. aeruginosa meningitis are high. It occasionally occurs in the neonatal period, but is most often associated with neurosurgical operations, especially when an indwelling intracranial catheter is inserted. The organism may occasionally be isolated from the cerebrospinal fluid (CSF) during fatal Pseudomonas septicaemia associated with the neutropenia induced by childhood leukaemia or its treatment (Hughes, 1971).

The case is now reported of a boy of 21 months who made a complete recovery from $P$. aeruginosa septicaemia and meningitis with neutropenia, before the diagnosis of hypogammaglobulinaemia was established or its treatment started.

\section{Case report}

S.G. is the only child of fit parents. He was born at term by normal delivery, and weighed $3.09 \mathrm{~kg}$. He was entirely breast fed for a month and subsequently given a cow's milk formula. He passed his milestones normally and he was immunized against diphtheria, pertussis, tetanus and poliomyelitis (Sabin vaccine) at 4 months, 7 months and 1 year of age without complications. Before his admission he had had occasional colds but no serious infection. There is no known family history of serious infection or malignant disease.

At 20 months of age he developed bronchitis for which he was given a tetracycline with amphotericin suspension. He was well after 4 days, but 2 days later his fever returned, with vomiting, cough and a large boil on his thigh. On admission his temperature was $39.5^{\circ} \mathrm{C}$; his tonsils were inflamed and the follicles were covered with a white exudate; and he had a necrotic indurated lesion $4 \mathrm{~cm}$ in diameter on the posterior aspect of his right thigh. There was mild cervical lymphadenopathy. No other abnormalities were found.

His peripheral blood total leucocyte count was $1.0 \times 10^{9} / 1$, and all the cells were lymphocytes. His haemoglobin was $8.4 \mathrm{~g} / \mathrm{dl}$, but platelets were plentiful. A chest X-ray showed middle lobe and lingular consolidation. $P$. aeruginosa was cultured from the thigh lesion and from a blood culture taken on admission. He was treated with intravenous carbenicillin $(660 \mathrm{mg} / \mathrm{kg}$ body-weight/day) and gentamicin $(6 \mathrm{mg} / \mathrm{kg} /$ day $)$.

His condition deteriorated over the next $24 \mathrm{hr}$ and he developed widespread macular lesions on the skin. Tobramycin $(5 \mathrm{mg} / \mathrm{kg} /$ day $)$ was substituted for gentamicin, on the basis of greater sensitivity in vitro of the Pseudomonas to this agent. A transfusion of 1.0 and $1.4 \times 10^{9}$ of $\mathrm{ABO}$-compatible leucocytes obtained from his father was infused on each of the next 2 days. After this he began to produce large numbers of his own neutrophils.

During the first 3 days of his illness he gradually became drowsy but he had no evidence of raised intracranial pressure or abnormal signs in the central nervous system. On the fourth day his CSF contained 1230 leucocytes/ $\mu 1$ ( $75 \%$ polymorphs), a protein of $200 \mathrm{mg} / \mathrm{dl}$, and glucose $13 \mathrm{mg} / \mathrm{dl}$. $P$. aeruginosa was isolated on culture. An unexplained transient asymptomatic thrombocytopenia occurring the day after intravenous tobramycin was started and 
lasting 4 days delayed the initiation of intrathecal gentamicin therapy which was begun on day 9 and was continued for the next 8 days ( $1 \mathrm{mg}$ daily). No bacterial growth was obtained from the CSF after intrathecal gentamicin had been started.

All antibiotics were discontinued 26 days after the start of treatment and he remained well, although his CSF at this time showed a persistent pleocytosis (66 leucocytes $/ \mu \mathrm{l})$ and a high protein $(100 \mathrm{mg} / \mathrm{dl})$. His chest X-ray also showed no change from the admission film. By 34 days from the start of his illness he was well enough for discharge, but he did not return to full normal health for at least 1 month after this. His chest X-ray remained abnormal showing persisting collapse/consolidation of the lingula and middle lobe until 6 months after his clinical recovery, although at no time did he have respiratory symptoms or clinical signs other than a mild cough.

Subsequent investigation of his immune status showed all immunoglobulin fractions to be severely depressed and anti-B isohaemagglutinin was virtually absent (blood being group A Rhesus negative). His absolute lymphocyte count was always normal; and a strong reaction was obtained after intradermal injection of Pseudomonas antigen, but not other antigens (Table 1), an indication that his cellmediated immunity was intact. A very low level of circulating antibody to the Pseudomonas was found in his serum. He has subsequently been treated with
TABLE 1. Investigation of immune status, performed at 2 years of age, 2 months after illness

\begin{tabular}{lrll}
\hline Immunoglobulins & Leucocytes & \\
IgG: $\quad 0.16 \mathrm{~g} / \mathrm{l}$ & Total: & $9.2 \times 10^{9} / 1$ \\
IgA: $\quad<0.10 \mathrm{~g} / 1$ & Neutrophils: & $4.8 \times 10^{9} / 1$ \\
IgM: $\quad<0.10 \mathrm{~g} / 1$ & Lymphocytes: & $3.7 \times 10^{9} / 1$
\end{tabular}

Estimation of ' $B$ ' lymphocytes (surface immunoglobulin method): $2 \%$ of total

Isohaemagglutinins: Blood group A Rh-ve. Just detectable anti-B

Antibodies against:

Pertussis immunization: CFT $<1: 5$ Agg $<1: 5$ HAI $<1: 5$

Polio (Sabin vaccine) all 3 types: CFT $<1: 8$

Serum complement:

$\mathrm{C}_{6}$ fraction: $0.55 \mathrm{~g} / \mathrm{l}$

$\mathrm{C}_{4}$ fraction: $0.50 \mathrm{~g} / 1$

Delayed hypersensitivity skin testing against:

Streptokinase/streptodornase: negative

Candida antigen: negative

Tuberculin PPD: negative

Pseudomonas antigen: positive

CFT, Complement fixation test; Agg, agglutination; HAI, haemagglutination inhibition.

weekly intramuscular gammaglobulin $250 \mathrm{mg}$, which has raised his serum IgG level to $2 \cdot 10 \mathrm{~g} / 1$ (measured 6 days after the second such injection). He has remained in good health and has grown normally in height and weight since beginning gammaglobulin therapy.

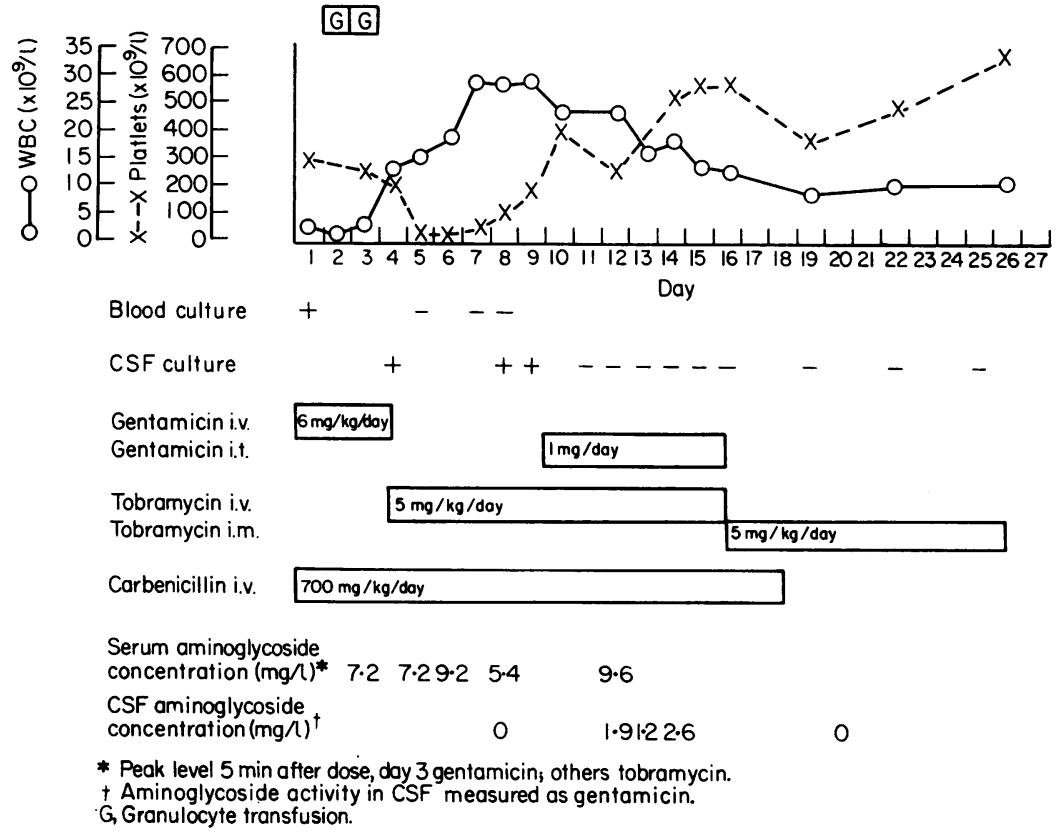

FIG. 1. Details of treatment and clinical and laboratory observations. 
Microbiological and immunological investigations

$P$. aeruginosa (serological type 4 , not typable by bacteriophages) was isolated from blood, CSF, the thigh lesion, and nose, throat and ear swabs. The isolate from blood (tested by the broth dilution technique, in Bacto heart infusion broth, with final inoculum of approx. $10^{4}$ colonies $/ \mathrm{ml}$ ) had minimal inhibitory concentrations: carbenicillin $50 \mathrm{mg} / \mathrm{l}$; gentamicin $1.2 \mathrm{mg} / \mathrm{l}$; tobramycin $0.15 \mathrm{mg} / 1$.

Antibiotic concentrations were estimated by an agar diffusion method employing Bacillus subtilis spores, after treatment of fluids with broad spectrum $\beta$-lactamase (Sabath et al., 1971). 'Peak' concentrations are shown in Fig. 1. In all instances when a serum tobramycin concentration was estimated before a dose was given, $6 \mathrm{hr}$ after a previous dose, very low values $(<0.5 \mathrm{mg} / \mathrm{l})$ were obtained. Serum taken on the eighth day was bactericidal to the $\boldsymbol{P}$. aeruginosa isolate at a dilution of $1: 16$.

The immunological investigations are set out in Table 1.

\section{Discussion}

The association of a low isohaemagglutinin level, a transient neutropenia and the observed hypogammaglobulinaemia in the presence of normal cellmediated immunity and absent plasma cells in the bone marrow, make the diagnosis of X-linked hypogammaglobulinaemia almost certain in this boy. Neutropenia occurs in $9.4 \%$ of children with this condition, normally in response to minor infections (Medical Research Council, 1971). In this patient there were three possible causes for his neutropenia. It could have resulted from his bronchitis, or from its treatment, since tetracyclines have been reported to cause bone marrow aplasia (De Gruchy, 1964). Pseudomonas infection itself is known to cause depression of granulopoiesis and platelet production in burned patients (Newsome and Eurenius, 1973). His haematological abnormalities were found to be associated with maturation arrest of the granulocyte precursors in the bone marrow at the myelocyte stage, with abnormal red cell precursors and depressed megakaryocyte numbers-appearances similar to those seen occasionally in virus- or druginduced bone marrow arrest. A second marrow sample 2 months later was normal, apart from virtual absence of plasma cells. There was no evidence of leukaemia in either of the two marrow samples. $\boldsymbol{P}$. aeruginosa may have become the infecting agent because of colonization by this resistant organism during tetracycline therapy. The lesion on the thigh may have been the primary source for the bacteraemia.

In leukaemia, $\boldsymbol{P}$. aeruginosa is a common cause of bacteraemia in phases of neutropenia either induced by the disease or its therapy. Recovery relates closely to haematological improvement. A high mortality and incidence of residual cerebral damage is reported in patients with Pseudomonas meningitis. The absence of specific replacement therapy for his undiagnosed hypogammaglobulinaemia makes this patient's recovery the more remarkable, although the spontaneous resumption of granulopoiesis was probably of paramount importance. The patient was initially treated with intravenous carbenicillin and gentamicin, and later with carbenicillin and tobramycin. Blood cultures were always negative after this treatment had been started; the regimens gave satisfactory peak gentamicin and tobramycin concentrations and a bactericidal activity of serum at a dilution of $1: 16$. Tobramycin and gentamicin penetrate poorly into the CSF, even when the meninges are inflamed, and on the ninth day of treatment $\boldsymbol{P}$. aeruginosa could be cultured from the CSF and there was no detectable tobramycin present. After daily intrathecal gentamicin $1 \mathrm{mg}$ was begun, the CSF was always sterile on culture; and, at the time when the next intrathecal dose of gentamicin was administered, there were always present in the CSF concentrations of aminoglycoside antibiotics (assayed as gentamicin) at least equal to the minimum inhibitory concentration of gentamicin for the Pseudomonas. These results were achieved with a comparatively low dose of gentamicin; it has been suggested that $8 \mathrm{mg} /$ day should be given to obtain satisfactory results in adults (Rahal, Simberkoff and Rubinstein, 1974; Turgeon, Laverdiere and Perron, 1975).

There are no firm guide lines at present for the use of leucocyte transfusions, although the authors believe that their patient's deteriorating condition justified their use. Aminoglycoside antibiotics are thought to be less effective in neutropenic states. Dale et al. (1974) attempted to assess the value of granulocyte transfusion in experimental $\boldsymbol{P}$. aeruginosa pneumonia in dogs rendered neutropenic by total body irradiation. They were given either gentamicin alone or gentamicin and granulocyte transfusion. Viable bacteria were found at postmortem examination in dogs given gentamicin alone but not in granulocyte-transfused dogs, and in the latter group endotoxaemia was also reduced and survival prolonged significantly. Graw et al. (1972) from the same centre showed that in neutropenic leukaemic adult patients cure of septicaemia is significantly more likely when leucocyte transfusions are given on four consecutive days. There seems little doubt of the value of this addition to therapy, and it may have contributed significantly to S.G.'s survival, although it did not prevent spread of the infection to the meninges. The normal fresh plasma in which the white cells were suspended may also have contributed to his survival. 
Acknowledgments

We thank Dr D. C. L. Savage for advice and encouragement in the preparation of this report, and Dr V. G. Alder, Dr F. Carswell, Dr I. D. Fraser, Dr M. T. Parker and Dr G. L. Scott for assistance with the investigation and management of the patient.

\section{References}

Dale, D.C., Reynolds, H.Y., Pennington, J.E., Elin, R.J., PITTS, T.W. \& GRaW, R.G., Jr (1974) Granulocyte transfusion therapy of experimental Pseudomonas pneumonia. Journal of Clinical Investigation, 54, 664.

De GruChy, G.C. (1964) Clinical Haematology in Medical Practice, 2nd edn, p. 338. Blackwell Scientific Publications, Oxford.

Graw, R.G., Herzig, G., Perry, S \& Henderson, E.S. (1972) Normal granulocyte transfusion therapy. Treatment of septicemia due to Gram-negative bacteria. New England Journal of Medicine, 287, 367.

Hughes, W.T. (1971) Fatal infections in childhood leukemia. American Journal of Diseases of Childhood, 122, 283.

Medical ReSEARCH CounCIL (1971) Hypogammaglobuli- naemia in the United Kingdom. MCR Special Report Series No. 130: London.

Newsome, T.W. \& Eurenius, K. (1973) Suppression of granulocyte and platelet production by Pseudomonas burn wound infection. Surgery, Gynecology and Obstetrics, 136, 375.

Rahal, J.J., Simberkoff, M.S. \& Rubinstein, E. (1974) Intrathecal gentamicin. New England Journal of Medicine, 291, 533.

RoSEN, F.S. \& JANEWAY, C.A. (1966) The gammaglobulins. III. The antibody deficiency syndromes. New England Journal of Medicine, 275, 709.

Sabath, L.D., Casey, J.I., Ruch, P.A., Stumpf, L.L. \& FINLAND, M. (1971) Rapid microassay of gentamicin, kanamycin, neomycin, streptomycin and vancomycin in serum or plasma. Journal of Laboratory and Clinical Medicine, 78, 457.

Speirs, C.F., SelwyN, S. \& Nicholson, D.N. (1963) Hypogammaglobulinaemia presenting as pseudomonas septicaemia. Lancet, ii, 710.

Turgeon, P.L., Laverdiere, M. \& Perron, L. (1975) Successful treatment of Pseudomonas meningitis and septicemia in a leukemic neutropenic adult. American Journal of Clinical Pathology, 63, 135.

\section{Chronic rhinocerebral phycomycosis in association with diabetes}

\author{
JULIAN E. FERSTENFELD \\ M.D. \\ HAROLD D. Rose \\ M.D.
}

\author{
Steven H. COHEN \\ M.D.
}

\author{
Michael W. Rytel \\ M.D.
}

Section of Infectious Diseases, Department of Medicine, The Medical College of Wisconsin, Milwaukee County General Hospital, 8700 W. Wisconsin Ave., Milwaukee, Wisconsin 53226, U.S.A.

\section{Summary}

Two patients with rhinocerebral phycomycosis associated with diabetes are presented. The chronic nature of the illness in these two cases is emphasized in contrast to the more fulminant course of most previously reported patients. A high index of suspicion is needed to establish the diagnosis as rapidly as possible. Treatment of the underlying disease combined with amphotericin B therapy and radical surgical excision of all infected tissue is important to establish cure of these patients.

\section{Introduction}

Phycomycosis is a fungal infection caused by organisms belonging to the class Phycomycetes. Clinical infection with these fungi is found in association with pre-existing diseases such as diabetic ketoacidosis, leukaemia and lymphoma, uraemia, cirrhosis, septic abortion, and treatment with steroids and antibiotics. The infection can present clinically in several localized forms such as rhinocerebral, pulmonary or gastrointestinal, or may be seen in a disseminated form (Abramson, Wilson and Arky, 1967; Battock et al., 1968; Best et al., 1971; Gass, 1961; Gregory, Golden and Haymaker, 1943; Helderman, Cooper and Mann, 1974; Hoagland et al., 1961; McBride, Corson and Dammin, 1960; Meyer, Rosen and Armstrong, 1972; Medoff and Kobayashi, 1972; Sander et al., 1971; Straatsma, Zimmermal and Gass, 1962; Touche, Sutherland and Telling, 1963; Wasserman, Shields and Sporn, 1961). The rhinocerebral form is seen most often in association with diabetic ketoacidosis with $75 \%$ of all reported cases having such an association (Battock et al., 1968). Phycomycosis of the rhinocerebral type usually presents as a progressive disease with a fulminant course. Despite present therapy, death often occurs within a few days to several weeks after 